\title{
SURVEYING AND COMPARING THE ARCO DEI GAVI AND ITS HISTORICAL WOODEN MAQUETTE
}

\author{
Francesco GUERRA, Paolo VERNIER \\ Università IUAV di Venezia, SdL Laboratorio di fotogrammetria, Italia \\ guerra2@iuav.it; vernier@iuav.it
}

Keywords: laser scanning, 3D modelling, digital photogrammetry, cultural heritage, virtual anastylosis, preservation, documentation.

\begin{abstract}
Actually geometrics' science offers new opportunities and interesting applications in the field of Cultural Heritage. These applications are strictly related to preservation, restoration but even to cataloging and reproducing a monument that no longer has its original integrity.

The possibility of obtaining 3D data, of such a model close to reality, enables us to realize studies that sometimes are too complex or impossible. The paper will describe the study of a monumental arch, the Arco dei Gavi, built in Verona during the I sec. A.C., that was destroyed in 1805 by the Napoleonic army, and its wooden model that was realized in 1813 and it has a very important role concerning the monument's reconstruction. The purpose is to realize two threedimensional models which can be comparable to each other, two models with recognizable differences, similarities and discontinuities about shapes and single elements that compose the monument. It should also be noted that some original parts of the monument have not been relocated but are badly preserved in a museum: the 3D digital model helps to identify these parts in their original location. The main steps of the work can be summarized in:

- collecting the historical documentation of Arco dei Gavi and its representations;

- $\quad$ identifying proper instruments (laser scanning and photogrammetric hardware and software);

- $\quad$ surveying the Arch and its wooden model;

- $\quad$ identifying a unique and shared reference system;

- comparing both digital models related to the same scale;

- choosing a three-dimensional representation to emphasize the results;

- reallocation of outstanding pieces (virtual anastylosis).
\end{abstract}

\section{INTRODUCTION}

Thanks to ever-advancing software and hardware tools, modern Geomatics offers new, interesting applications in the cultural heritage field. These applications do not just pertain to the preservation, restoration and reproductionprototyping of monuments. They also deal with the digital, virtual planning of possible structural and conservative interventions on monuments that have been demolished or reconstructed, as well as with the cataloguing and studying of objects of various dimensions. The possibility of obtaining a digital 3D model, at once faithful to reality and untied from its strict bounds, enables us to perform operations and analyses otherwise too complex or impossible. Scale models and maquettes evoked a high level of craftsmanship and their perfect details had the effect of crystallizing ideas and anticipating the future. They aided the architect in "knowing the beauty of a building, whose idea he just conceived, before even starting its construction." We examined the Arco dei Gavi (Fig. 1), an architectural monument dated I century a.C., which was demolished in 1805; and its wooden model (Fig. 2), built in 1813, which had a crucial role in the monument's reconstruction. In the case under study, the wooden model has a crucial, specific function, namely, it embodies the database to use for the monument's reconstruction, as well as the tridimensional historic memory of a structure that has been demolished. Hence, it will no longer anticipate the future, but it will help the architect to bring the monument back to life. The wooden model was made by a skilled carver, Sughi, based on the outcomes of a direct survey of the arch by architect Barbieri and from previous surveys by Palladio and architect Ederle. In 1932, the monument was rebuilt starting from this model, thanks to the intervention of professor Avena, director of the city museums at the time. Today the numerical, digital model enables us to include all possible "views" in a single representational system and guarantees the same functions of the iconic, diagrammatic and mathematical models. The use of new architectural survey technologies produces a great amount of data that need to be computed in order to create significant and specific digital representations. Any form of representation, such as points or surfaces, has to refer to its generating element, namely, its measure. Every model can be classified depending on the degree of adherence to its original data. Therefore, the numerical model has to adhere as much as possible to the arch and its maquette, in order to compare them without incurring deceptive simplifications or interpretations. 

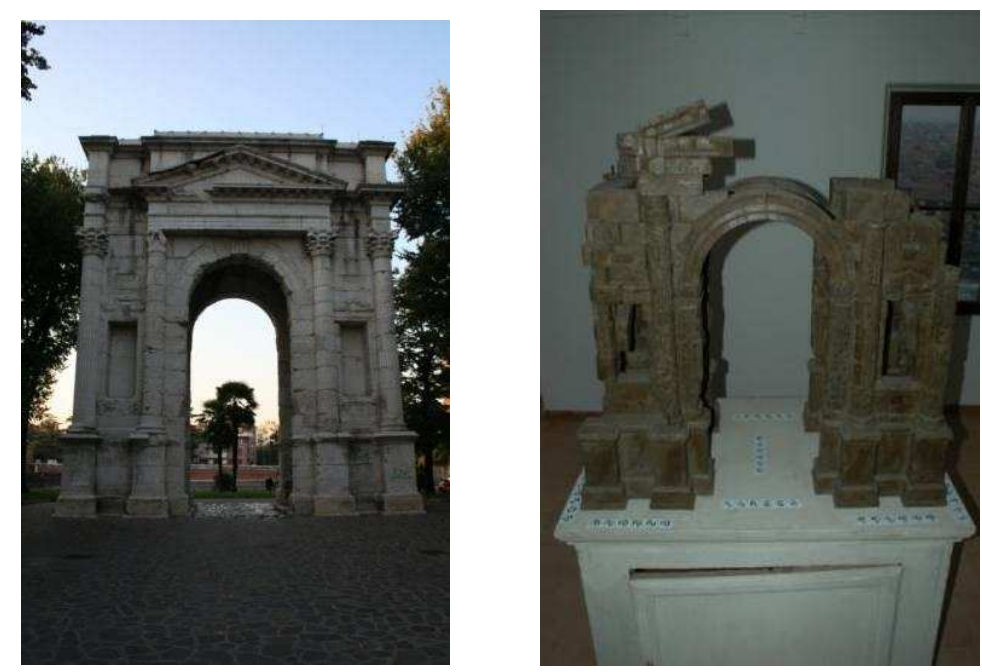

Figure.1,2 Arco dei Gavi and its wooden maquette.

\section{OBJECTIVES}

Studying these structures, the elements that raised our interest the most were their creation and their story. In theory, they should be the scale representation of the same object, of the same "planning idea." However, in this particular case the sequence of events is inverted. The purpose of building a wooden model, a scale model, or a digital model is to make tangible a project otherwise too difficult or complex to understand. In this case instead, we have the scale representation of a previously existing monument. In the course of centuries, the monument has been used, modified, surveyed by notable architects such as Serlio (Fig. 3) and Palladio (Fig. 4), and finally demolished. Hence, what we want to restore and preserve in time is the original aspect of the arch, by building a faithful scale reproduction of the monument. Architect Barbieri surveyed every single piece of the arch soon after its demolition and hypothesized a reconstruction based on Palladio's survey, dated 1500, and prof. Ederle's survey, dated shortly before its demolition. Thus, the goal is to obtain two numerical models to compare and analyze; two models that enable us to identify differences, equalities and discontinuities in shape and in its composing pieces.
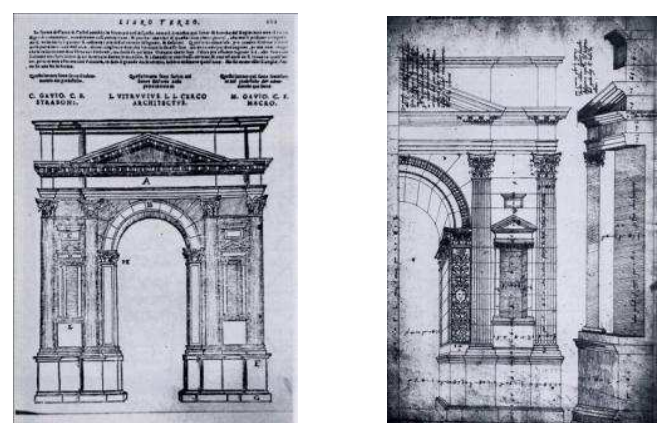

Figure 3;4. Serlio's and Palladio's drawings.

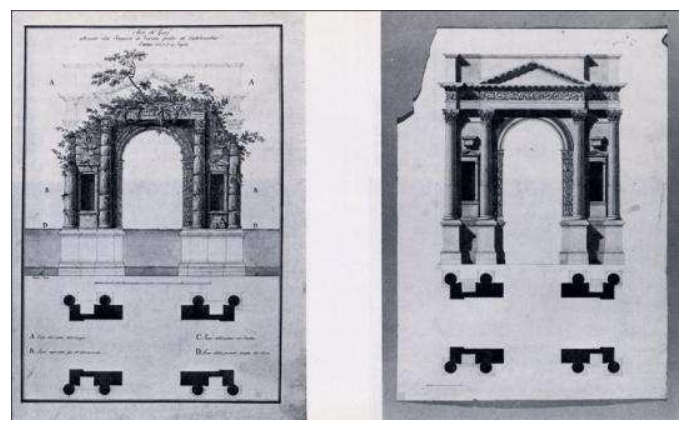

Figure 5; 6. Prof. Ederle's reconstructive hypothesis

Thus, the goal is to obtain two numerical models to compare and analyze; two models that enable us to identify differences, equalities and discontinuities in shape and in its composing pieces. Obviously, the choice of the equipment used to perform the survey on two objects so different in structure from one another becomes critical. In fact, the wooden model is scale 1:10 (calculated 1:9.7) compared to the original. Therefore, it is necessary to use two instruments that give comparable outcomes once compared on the same scale. The two instruments we identified are: the terrestrial laser scanner system RIEGL LMS-Z390i with an interfaced metric aerial camera, which enables us to obtain textured triangulated surfaces and 3D orthophotos; and ScanSystem ScanProbe LT for the survey on the wooden model. The precise distance measurement of the first instrument is $\pm 4 \mathrm{~mm}$ on a single scan and decreases up to \pm 2 on a scansequence. The precision of the second instrument ranges between 0.1 and $0.2 \mathrm{~mm}$ depending on the calibration. 


\section{THE SURVEY OF THE ARCO DEI GAVI USING TERRESTRIAL LASER SCANNER SYSTEM LMS-Z390I}

We planned three scans for each front side and two for the lateral sides. The inner and covered areas of the arch had to be scanned 4/5 times, changing the vertical axis of the instrument, in order to adequately acquire the whole area (Fig.8,9). Then, we proceeded with the materialization on the ground of two landmarks to realize a topographic base from which to survey the supports for the registration of the clouds. In fact, the object and the surrounding area have been marked with about 25 reflective targets, needed to record the scans in the same reference system. The next step was to identify the area of the first "cloud" of the area to survey and to define the resolution of the laser. The exit angle between an emission and the next will provide a geometric spacing on the object. More precisely, it will provide the means of this spacing. The distance of the various surfaces and their angle relative to the equipment will affect the spacing between two points. The angular value calculated on the point of maximum distance, 0.02 deg., allowed us to obtain a point every 2-3 mm. With these initial parameters we obtained scans made of about 10,000,000 points each. After the metric measurements, we acquired the images with the camera mounted on the laser system. The known position of the digital camera and of the camera perspective centre, the focal length of the lens and the parameters to correct lens distortions constitute a calibrated camera, which provides images with known inner and outer orientation. The clouds' recording procedure establishes a connection between the coordinates of the target, acquired with the laser, with those acquired using the topographic method. This method calculates the least squares on the distances between points to minimize the error and it also automatically recalculates the position matrix and the laser's orientation. The computation's outcome is a standard deviation between 2 and $4 \mathrm{~mm}$ on the merging of each individual cloud with the topographic points. After the orientation, we proceeded with the clouds' cleaning with filters to eliminate the outliers, the noise and any other interfering element that could invalidate or "spoil" the metric data. Furthermore, we operated a controlled decimation to reduce the data by using the octree algorithm with a resolution close to $5 \mathrm{~mm}$, so that we were sure not to eliminate points there were important for the geometrical understanding of the arch.
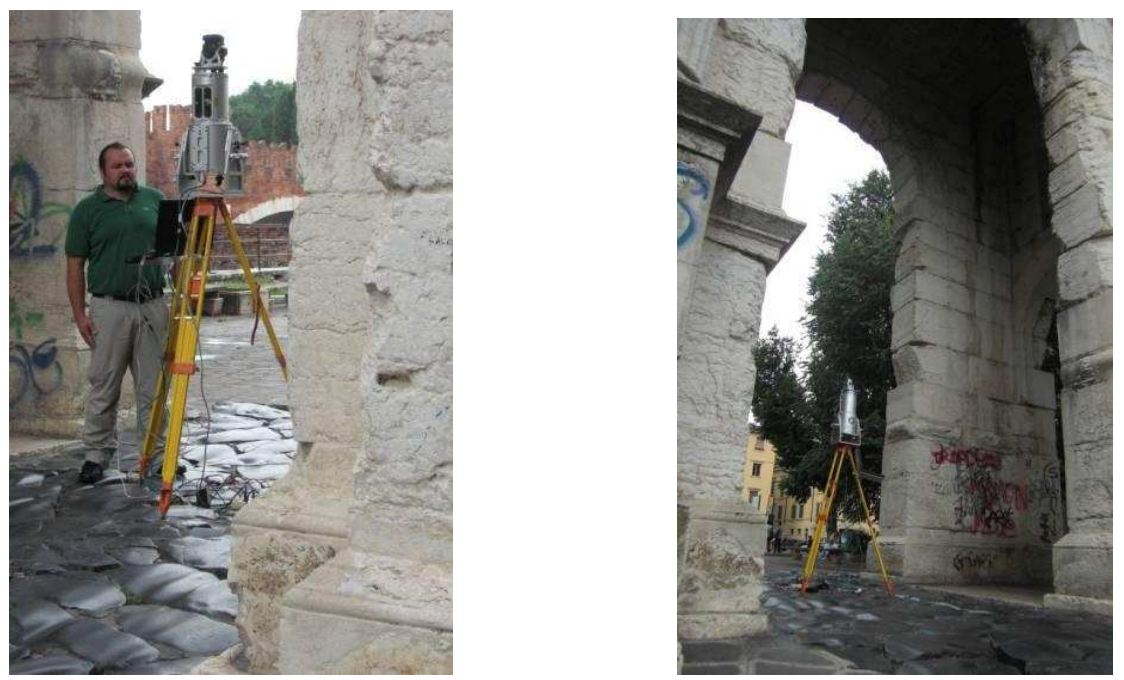

Figure 8;9. Recording with laser scanner of the inner and covered area of the arch
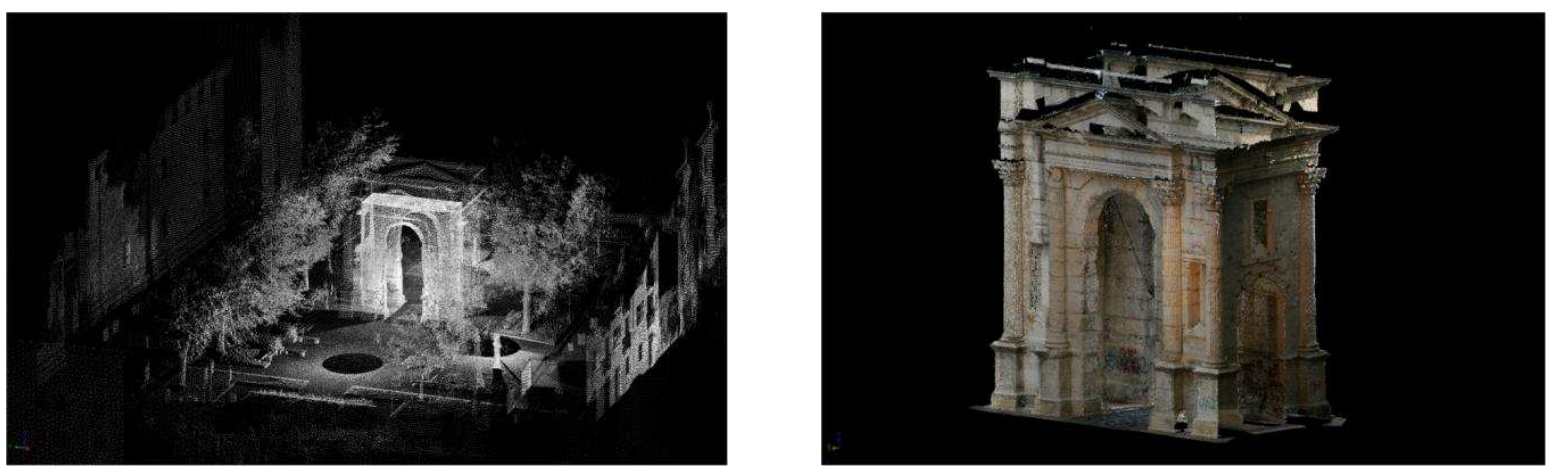

Figure 10;11. View from the top of the merging of the clouds 
After the clouds' cleaning, to which we attributed the RGB value (Fig.10,11), and after their merging, we obtained the first 3D data for the comparison, in other words a tridimensional model for surfaces, the mesh (Fig.12). We did not fill the gaps in the mesh caused by shadowed areas, to prevent parts not corresponding to reality to invalidate the analyses and the comparison of the wooden model with the mesh. The metric data obtained served also to reconstruct a simplified model of the arch in a CAD environment to obtain a digital database for each individual piece (still under processing).

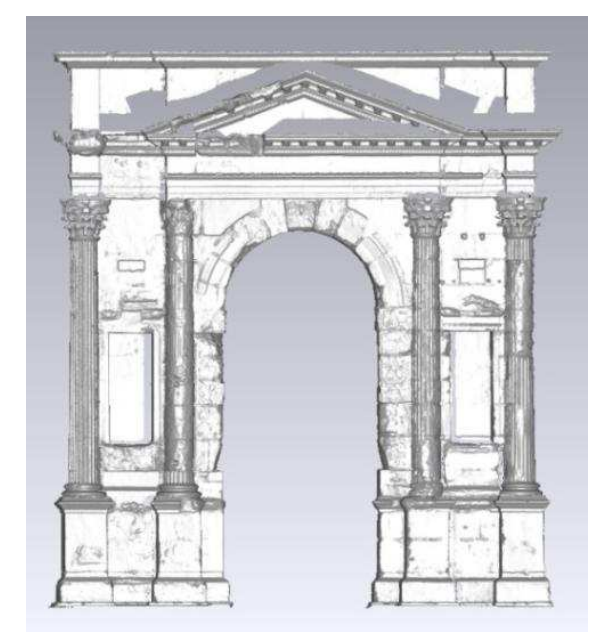

Fig.12 Mesh of the facade (RapidForm2006)

\section{THE SURVEY ON THE MAQUETTE OF THE ARCO DEI GAVI WITH DIGITAL SCANNER SCANPROBE LT}

The 3D triangulation system used projects onto the object under study eight patterns at increasing frequency, that is, images composed by alternating black and white vertical stripes that warp depending on the surface surveyed. The video camera records the images. Projector, video camera and pattern's points represent the vertices of a triangle in the space and, therefore, the spatial position of the points on the profile is derived according to the known triangulation method. For each point, we also recorded the radiometric values of the RGB channels. The system needs to be calibrated after every change in the geometric configuration, or rather in the baseline, which is the distance between sensor and projector. Calibration procedures consist of the acquisition of a panel of known geometry, performed from different points, and provide the parameters of the inner and outer orientation of both sensor and projector. Knowing these parameters is critical for an accurate triangulation. Since this instrument enables us to vary the optogeometric setup, we can modify the baseline's distance depending on the special requirements that each object has in terms of measuring. First, we defined the recording distance in function of the measuring area and the desired points' density on the object. In our case, dimensions were up to $1 \times 1 \mathrm{~m}$ and we wanted to obtain highly dense, detailed data to align the clouds, used as control point. To do so, we chose a calibration that allowed the recording of 400x300 mm areas from a distance of $70 \mathrm{~cm}$. With these parameters, accuracy reaches a tenth of a millimeter. Once established the system's settings and the number of recordings, we started with the acquisition of the "range map" (that is, a 3D image made out of thousands of points' coordinates), each one describing a single portion of the object's surface (Fig.13,14). After this approximate alignment, we performed an alignment using the ICP (Iterative Closest Point) algorithm, which entails the iterative minimization of the distance between two discretized surfaces. Once all the range maps were properly aligned, we proceeded with their merging to generate a single mesh made of polygons. We could then refine the mesh with editing techniques, such as, smoothing and other filters needed to utilize the data. At this point, it was possible to run direct measurements and analyses, in order to verify if the outcome accurately reproduced the geometric shape of the object acquired. To survey the wooden model, we had to perform about 150 scans, each made by 500,000 points. Structure of the object, quality of the scanning and accuracy of the survey required an higher level of control over the recording of the clouds in the same reference system. We used the ICP algorithm implemented on Geomagic 10 software. We also checked the recording, to avoid the drift problem (Beraldin, 2004), by creating a photogrammetric support using ImageModeler 2009, a software for monoscopic digital photogrammetry. To check the recording we created a reference system and a points' set: first we performed the scanning with ICP, creating a set of swaths (8 swaths, two for each side, made of 20 scans each); then we recorded and checked these swaths thanks to the photogrammetric control points (Fig.15). 

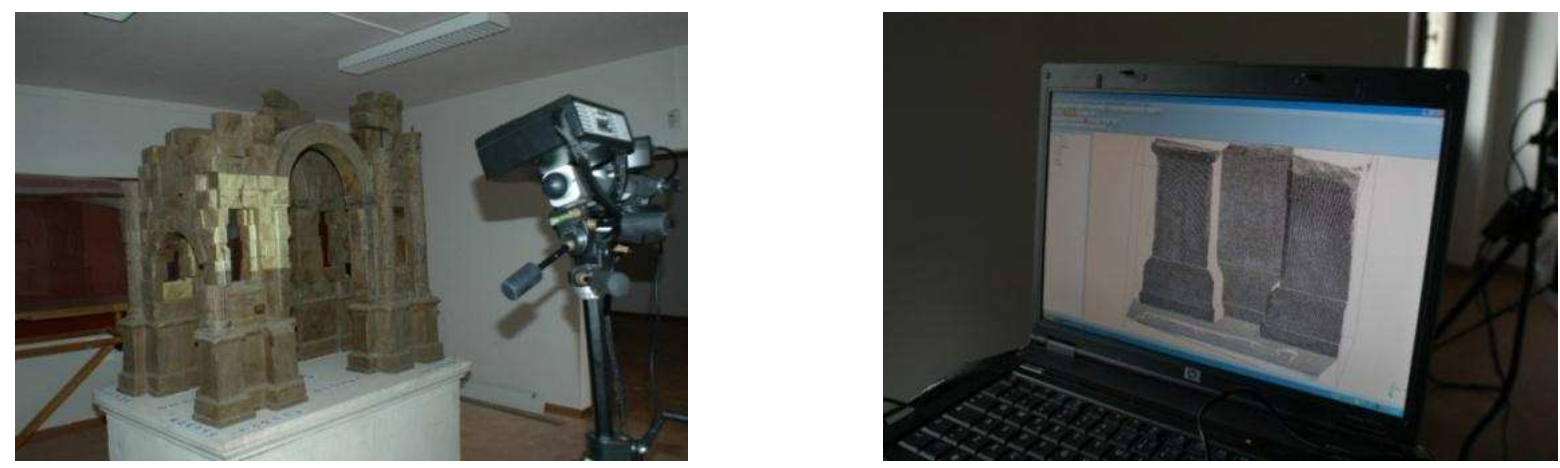

Figure 13;14. Recording phase and subsequent data display.

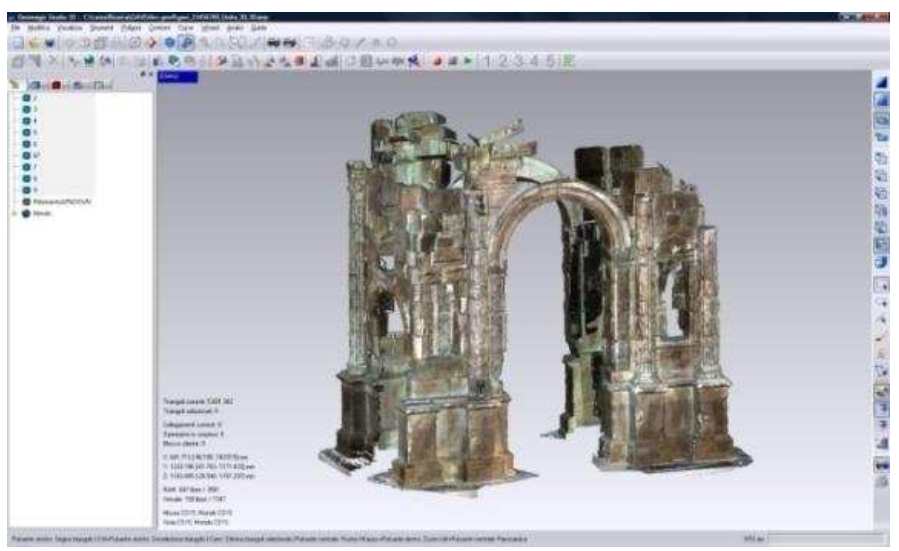

Figure 15. Complete merging thanks to the control points of the photogrammetric support

Purposely, during the editing phases we did not fill the gaps due to a lack of data. We did not want to add computed areas to the real surface of the surveyed object, because these would have been misleading during the subsequent comparisons and analyses between the two facades. Therefore, the outcome was a model for surfaces of most of the wooden arch, textured on the RGB value, which was measurable and comparable. The inner part has not been acquired due to the excessive dimensions of the 3D scanner.

\section{ANALYSES AND COMPARISON}

After the acquisition of the data and the first phase of post-processing, during which we filtered and cleaned the clouds and we recorded in the same reference system, and after the possible assignation of RGB data, we started the preanalyses phase. The aim of this phase was to verify the compatibility and comparability of the data. Before the direct comparison of the models, we did some measurements of the distance on single clouds and on sets of recorded clouds, in order to understand if the two models were similar, geometrical descriptions of the same object. Measuring the arch's narrow areas as well as its whole height and width, that is to say invariant data concerning macro and micro areas, enables us to obtain the first valid and encouraging results. In fact, the differences between the measures on the arch and the model were in the vicinity of 1-2 cm. This represents an acceptable difference for a nominal scale of 1:50-1:100 and it is in line with the equipment's accuracy and the estimated accuracy of the 3D model. After having verified the comparability, we proceeded with the triangulation of the data to obtain a 3D model for surfaces.

\begin{tabular}{|c|c|c|c|}
\hline & Architecture's Arch & Maquette & Difference \\
\hline height & $1,068 \mathrm{~m}$ & $1,070 \mathrm{~m}$ & $0,002 \mathrm{~m}$ \\
\hline width & $1,098 \mathrm{~m}$ & $1,097 \mathrm{~m}$ & $0,001 \mathrm{~m}$ \\
\hline base's width & $0,307 \mathrm{~m}$ & $0,306 \mathrm{~m}$ & $0,001 \mathrm{~m}$ \\
\hline
\end{tabular}


Geomagic 10, which is the same software we used for the recordings with ICP algorithm of the triangulation scan, has been used to shift from the model for points to the mesh. As an initial area to use for the analysis and the comparison, we chose the facade facing the city which, according to the historical references to it in literature, was also originally facing the old city. The choice was due to the fact that this part offers the highest number of original pieces, including some parts of the tympanum, which is otherwise completely reconstructed. One of the tympanum pieces is not even represented in the wooden model. Most likely, architect Barbieri did not survey this piece, because it is not represented in his drawings, but has been retrieved afterwards during the reconstruction phase. Once having eliminated all the points not belonging to the object, as well as the areas where the data were too noisy due to excessive distance or proximity to the CCD sensor, we calculated and built the mesh of the wooden model. After having cleaned the merging of the three scanning of the architecture, we created a second model for surfaces for the comparison (Fig. 16). The reference system defined by the photogrammetric support for the scanning of the wooden model has been used as the sole reference system to use to orient and compare the models for surfaces of the two objects.
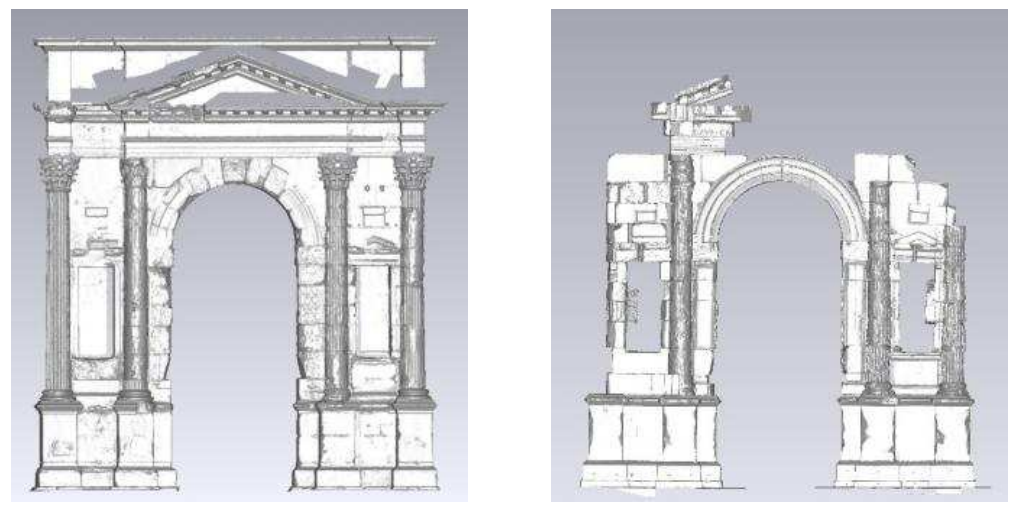

Fig.16: Mesh of the two facades.

We calculated the reference plane with ImageModeler2009, the monoscopic photogrammetric software, using 12 images, acquired with digital camera D100 20mm with a 6mp CMOS sensor, and employing a series of targets. On this plane we physically rested the wooden model and we calculated the Cartesian tern as well as a series of points in common between the maquette and the actual arch. Since the maquette is solely based on the reproduction of the antique blocks, the points only belong to the parts also present in the arch. Therefore, we were able to keep the 3D model of the maquette steady, using as a reference system the one we used for its orientation, and to operate a rototranslation in space with scale variation only of the 3D model of the architecture's arch. Effectively, we imported the reference system in the form of tridimensional coordinates using Geomagic10, software used for the management of 3D data and for rapid prototyping. On the same file, we imported the mesh of the arch's facade, which had been oriented by assigning to the architectural points, manually selected, the computed coordinates after the scale variation.

\subsection{Comparison between surfaces and grid of points}

The first comparison of selected macro areas highlighted the differences, corresponding to the areas that were completely reconstructed and those with some original pieces, and the overlaps. Analyzing piece by piece, we noticed how some elements have been replaced due to apparent deterioration, while in some other areas there are original pieces that are not featured in the wooden model. In 1932, the reconstruction phase of the attic, of some columns, of nearly the whole pediment, and of other areas of the arch, aimed to differentiate the new from the original, simplifying the details of mouldings, capitals and columns. This reconstructing strategy was very effective because it preserved the original parts, as shown by the laser data. Parallel to the comparison for surfaces in the same reference system, we also compared the two surfaces considering the scale variation and applied the ICP algorithm to the two geometries. This kind of comparison has been applied to the meshes of the facades using Geomagic10 software. We also compared the points' grid by overlapping the two grids with Surfer 9 software (Fig.17). In this case, the data did not undergo procedures that could possibly introduce geometric errors. The outcomes of the two subsequent comparisons supported the geometric proximity of the two objects, even if the direct comparison of the surfaces raised some problems related to substantial gaps in the data, due to a lack of data in the maquette compared to the reconstruction. In the case we modified the mesh of the architectural structure, eliminating all the new parts that had no correspondence with the data of the comparison, in order to have the same surfaces. To find a comprehensive representation of the monument able to emphasize the differences between reconstructed and original parts, we adopted two different 3D modelling methods for the two parts. With the modelling software Rhinoceros 4.0, we modelled the reconstructed parts, for example 
pediment and attic, by extrusion and revolution, two traditional 3D CAD modelling techniques. First, we drew the moulding profiles as well as the profiles of other architectonic details, and then we proceeded with the modelling of the surfaces. Next, we selected, cut and eliminated from the facade's mesh the new areas. The remaining data corresponded to the mesh of the original part, generated starting from the cloud of points describing the historical parts. This representation of 3D models, generated with a double procedure, highlighted even more the simplified areas compared to the recovered ones (Fig.18), and offered the possibility to interact directly on the digital product.
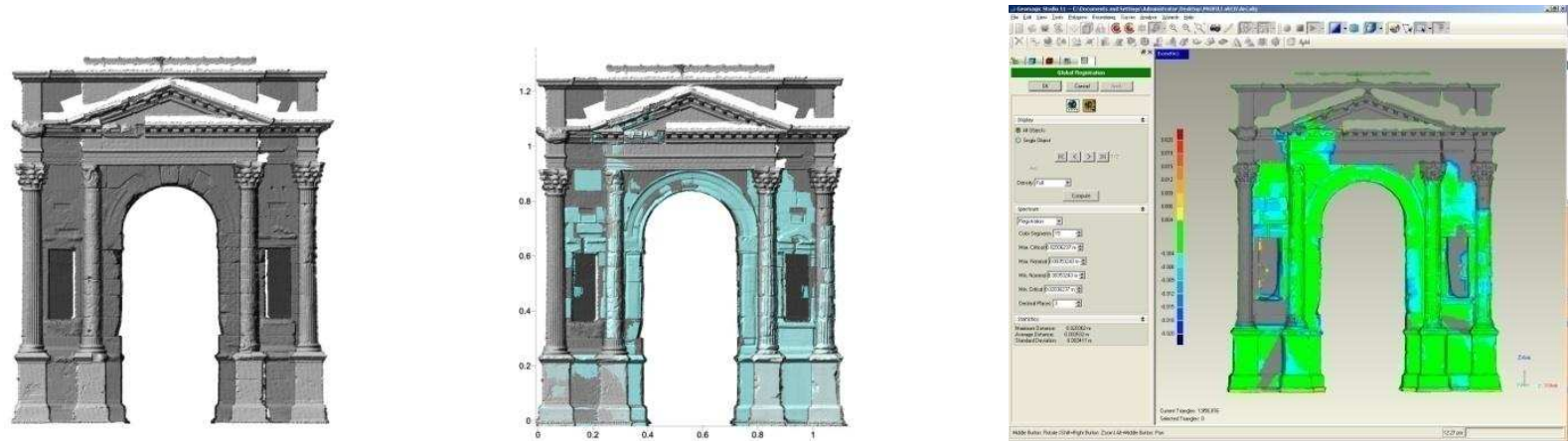

Figure 17. Overlapping of the two grids in Surfer and their subtraction in Geomagic.

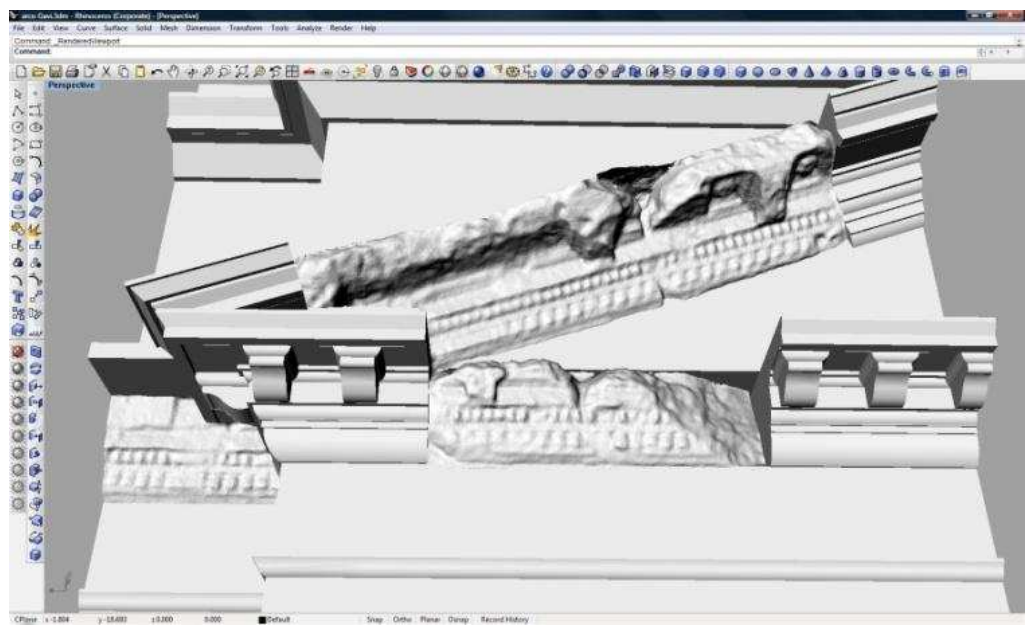

Figure 18. Detail of the 3D modelled with the mesh of the historical parts

\section{VIRTUAL RECONSTRUCTION}

An additional analysis concerned the digital reconstruction based on the tridimensional survey of the original pieces of the arch that have not been used for its actual reconstruction. Literature showed evidence of a piece of cornice of the attic found in 1960 during some digging operations and currently displayed inside Castelvecchio's garden. This finding shows the wrong reconstruction of the top part of the monument. The digital anastylosis consisted of the creation of a $3 \mathrm{D}$ model and in its positioning on the complete 3D of the arch and its purpose are the reconstruction of the arch and the evaluation of the objects' compatibility. For the angle of the cornuce however, the method used has been more significant and illustrative. The attic has eight edges and the drawing of the piece that has been found matches four of them. To insert and relocate the piece, we performed a roto-translation in space with the same scale. The data have the same scale because they belong to the architectural structure and have been acquired using the same software, Geomagic10. We uploaded the mesh of the whole facade in the orientation module, as well as the 3D for surfaces of the attic's angle. We kept the facade steady in order to have as a reference system the topographic support, ensuring its vertical positioning. We identified some homologous points, even if this operation presented some challenges because the reconstructed part has been quite simplified, while the piece found presents a rich moulding framework. Then, we virtually positioned the piece in its original location. It is evident that its positioning has been approximate and that metric and historic controls were weak. However, this can be the first step toward the restoration of the monument. 
Successively, the 3D data have been imported onto Rhinoceros 4.0, where we integrated modelling and mesh, to relocate it in its hypothetical original position (Fig.19). Once again, we want to stress the difference between the reconstructed ideal piece and the actual one that has been recovered.

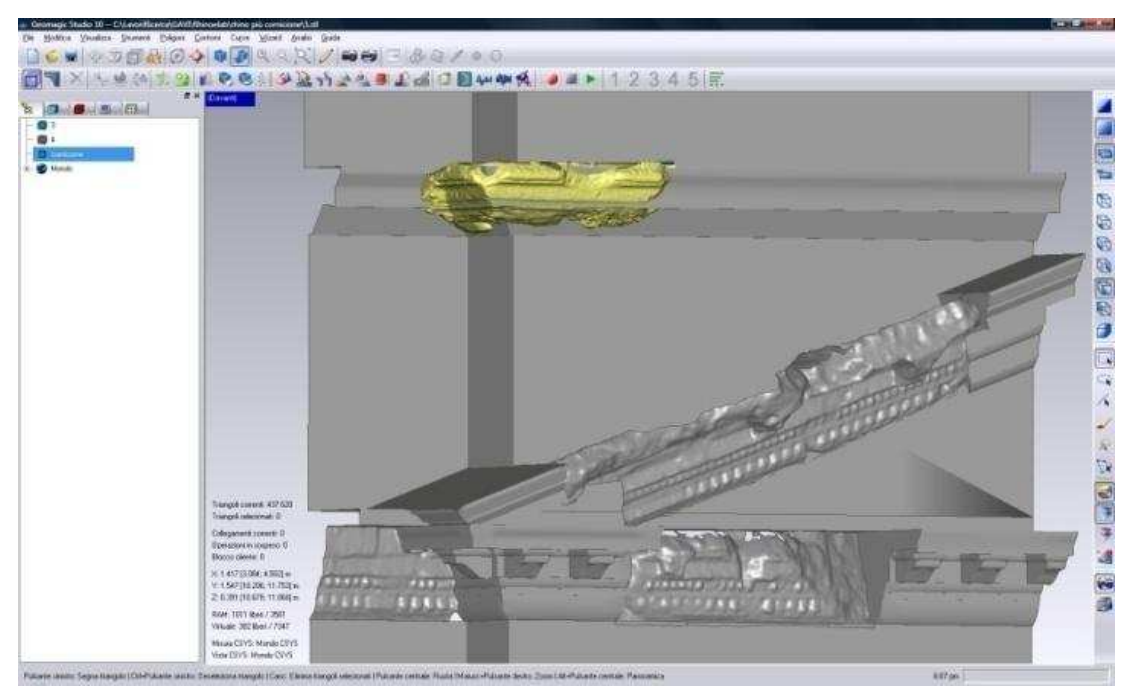

Fig. 19: Arch reconstruction

\section{CONCLUSIONS AND PERSPECTIVES}

We can state that the methodology's and instrument's choices permitted us to obtain analyzable and comparable tridimensional data. Also, software used allowed and, in certain cases, simplified modelling and comparison procedures. The choice to operate on the data grids as well as the use of a 3D mesh representation enabled us to work on the single surfaces, facade by facade, verifying not only the "vicinity" of the two objects according to macro areas, but also per single piece. The integration in the 3D for surfaces of modelled parts, corresponding to the areas that have been reconstructed, with the mesh obtained from laser data, corresponding to the original parts, allowed the emphasis on the current state of the arch, best illustrating how stylized the reconstructed part is. Future perspectives might include the analysis of the whole arch and the use of 3D solid modelling, instead of 3D for surfaces, to operate block by block. In this case also, we would integrate the mesh, recreating a tridimensional database containing all the information needed to recognize and know each piece.

\section{REFERENCES}

[1] Adami A., Guerra F., Vernier P., 2007 "Laser scanner and architectural accuracy test" in Proceeding of Cipa 2007 XXI International Symposium "Anticipating the future of the cultural past" Atene

[2] Adami A., Gnesutta M., Vernier P. 2010,Dalla scansione laser al modello: il caso esemplare di San Francesco della Vigna, in Architettura delle facciate: le chiese di Palladio a Venezia. Nuovi rilievi, storia, materiali. Marsilio Editore

[3] Balletti C., Guerra F., Vernier P., Studnicka N., Riegl J., Orlandini S., 2004. Practical comparative evaluation of an integrated hybrid sensor based on Photogrammetry and Laser Scanning for Architectural Representation, in ISPRS, International Archives of Photogrammetry and Remote Sensing, Commission V, ISPRS XX Congress, Istanbul, Turchia.

[4] Bitelli G., Tini M.A., Vittuari L., 2000. Close-range photogrammetry, virtual reality and their intergration in archaeology. In Proceedings of XIXth Congress of International Archives of photogrammetry and remote sensing, pp 872-879, Amsterdam

[5] Boehler W. 2003 Comparing different laser scanners: features, resolution, accuracy. In CISM, International Centre for Mechanical Sciences, Udine.

[6] Guidi G., Beraldin J.A., 2004. Acquisizione 3D e modellazione poligonale. Dall'oggetto fisico al suo calco digitale, Edizioni Poli.Design, Milano. 